\title{
CIRCUMFERENTIAL PROXIMAL FEMORAL ALLOGRAFTS IN REVISION SURGERY ON TOTAL HIP ARTHROPLASTY: CASE REPORTS WITH A MINIMUM FOLLOW-UP OF 20 YEARS
}

Bruno Dutra Roos ${ }^{1}$, Milton Valdomiro Roos ${ }^{2}$, Antero Camisa Júnior ${ }^{1}$

\section{ABSTRACT}

Among the options for femoral reconstruction in total hip arthroplasty (THA) revision procedures, in cases of extensive circumferential defects, is the use of proximal femoral allografts. This technique makes it possible to correct the hip abductor mechanism and the leg length discrepancy, as well as presenting osteoconductive potential. The authors report the clinical and radiographic results from two cases of THA revision using this technique, with a minimum follow-up of 20 years.

Keywords - Arthroplasty, Replacement, Hip /methods; Femur/surgery; Transplantation, Homologous/adverse effects; Prosthesis Failure

\section{INTRODUCTION}

Revision surgery on total hip arthroplasty (THA) in cases of extensive femoral bone defects is an enormous challenge. In such cases, use of conventional surgical techniques does not produce satisfactory results ${ }^{(1-2)}$.

Many surgical treatment methods for overcoming this problem have been described in the literature, but there is still no consensus regarding the best technique to apply. Circumferential allografts of the proximal or distal femur, fragmented bone grafts in association with support screens, cortical structural bone grafts, endoprostheses and femoral nails with distal fixation are some of the alternatives that exist ${ }^{(1-3)}$.

Use of proximal femoral allografts allows correction of the hip abductor mechanism and leg length discrepancy, and also presents osteoconductive potential in that it enables gradual substitution by allografts of the ${ }^{(1-3)}$.

The aim of the present study was to report on two cases of femoral reconstruction secondary to loosening of THA, using a circumferential allograft from the proximal femur and cemented implantation, with a minimum follow-up of 20 years. In reviewing the literature, we did not find any case reports that had used this technique with similar follow-up.

\section{CASE REPORTS}

\section{Clinical case 1}

The patient was a 46-year-old woman with a complaint of intense pain in the right inguinal and thigh region during active movement of her leg, which had started eight months earlier and had worsened 15 days ago. The patient had undergone THA on the affected side 11 years earlier, and had not previously presented any symptoms.

On physical examination, it was seen that she was unable to walk with weight borne on the right leg because of her painful condition. There was a leg length discrepancy such that the affected side had become shortened by $3 \mathrm{~cm}$.

Radiographically, signs of loosening of the THA (Muller prosthesis) could be seen, with verticaliza-

1 - Hip Surgeon in the Hip Surgery and Reconstruction Group, Orthopedic Hospital of Passo Fundo (HOPF); Instructor in the Medical Residence Program on Orthopedics and Traumatology, HOPF, Passo Fundo, RS, Brazil.

2 - Head of the Hip Surgery and Reconstruction Group, HOPF; Head of the Medical Residence Program on Orthopedics and Traumatology, HOPF, Passo Fundo, RS, Brazil. Work performed at the Orthopedics Hospital of Passo Fundo, School of Medicine of the University of Passo Fundo, Passo Fundo, RS.

Correspondence: Av. Sete de Setembro 817, Centro, 99010-121 Passo Fundo, RS, Brazil. E-mail: brunodroos@gmail.com

Work received for publication: February 15, 2011; accepted for publication: June 22, 2011.

The authors declare that there was no conflict of interest in conducting this work 
tion of the cemented acetabular component. The femoral implant presented signs of loosening, and the proximal third of the femur was severely compromised. Proximal migration of the greater trochanter had occurred, and there was a comminuted periprosthetic fracture at the level of the prosthesis. From the AAOS classification ${ }^{(4)}$, the femoral defect was considered to be type IV (Figure 1). Laboratory tests on infection markers showed that these were within the normal range.

The patient underwent THA revision, consisting of revision of the cemented acetabular component by means of an uncemented implant, and use of a non-irradiated circumferential allograft from the proximal femur and a cemented femoral component, in association with stabilization of the graft with a plate and screws (Figure $2 \mathrm{~A}, \mathrm{~B}$ and $\mathrm{C}$ ).

In both cases, gentamicin in association with cefazolin was used as antibiotic prophylaxis for 10 days (during the hospital stay), and mechanical and medicational thromboembolic prophylaxis was administered for 30 days. After the operation, the patient was allowed to walk with the aid of crutches and without placing weight on the operated leg, from the $10^{\text {th }}$ day onwards. The patient was guided to do active hip

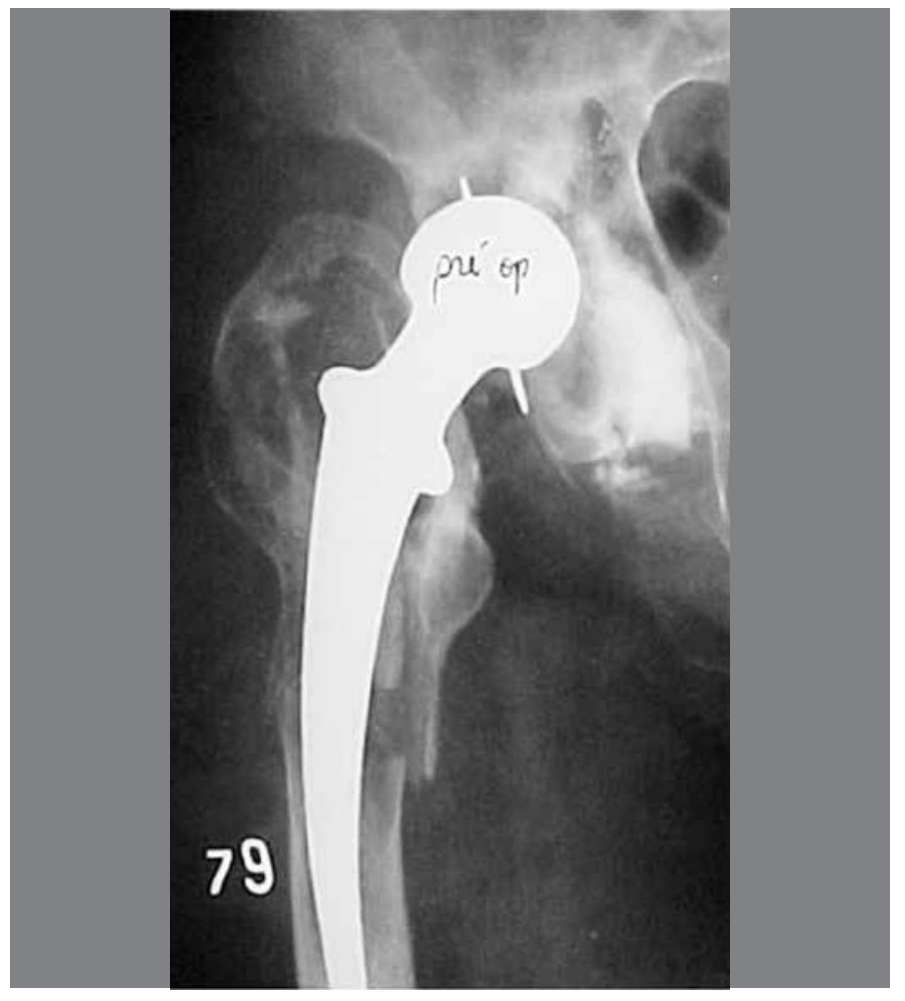

Figure 1 - Radiograph on 46-year-old female patient, showing signs of loosening of THA (Muller prosthesis), with AAOS type II acetabular defect and type IV femoral defect ${ }^{(4)}$.

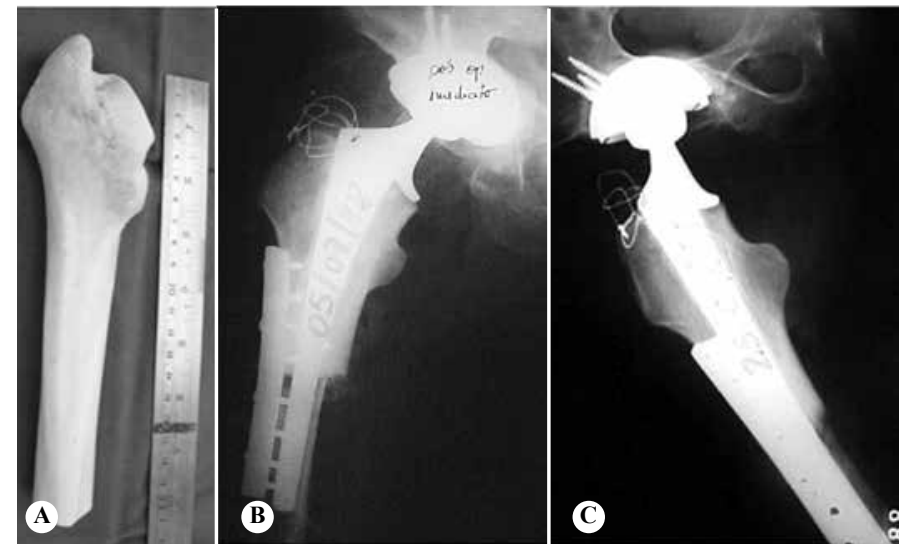

Figure 2 - (A) Circumferential proximal femoral allograft. (B) Anteroposterior (AP) radiograph produced during immediate postoperative period. (C) Lateral radiograph produced during immediate postoperative period.

abduction and flexion exercises, six weeks after the operation, and full weight-bearing on the leg was allowed three months after the operation.

After two years of postoperative follow-up, the patient's Harris hip score ${ }^{(5)}$ was 89 points. Radiographically, it was observed that the implant was stable, without signs of graft reabsorption. The allograft had consolidated at the graft-host junction and the cerclage threads had broken. After 12 years of postoperative follow-up, migration of the greater trochanter $(1 \mathrm{~cm})$ was observed radiographically. However, there was no change in the patient's gait pattern (Figure $3 \mathrm{~A}$, $\mathrm{B}, \mathrm{C}$ and D).

Today, after 21 years of postoperative follow-up, the patient's Harris hip score ${ }^{(5)}$ is 76 points. Radiographically, the implant is seen to be stable, without signs of graft reabsorption (Figure 4 and B).

\section{Clinical case 2}

The patient was a 66-year-old woman with a complaint of intense pain in her right thigh with active movement, which had started three weeks earlier. She did not have any history of trauma. She was unable to walk because of her painful condition. The patient reported that she had suffered a fracture of the femoral neck nine years earlier, for which she had been treated by means of partial hip arthroplasty and subsequently (six years afterwards), two new revisions of the implant.

On physical examination, leg length discrepancy was observed, with the right side shortened by $2 \mathrm{~cm}$. It was not possible to assess the range of motion of the affected hip, because of the intense pain.

Radiographically, a fracture due to fatigue of the 


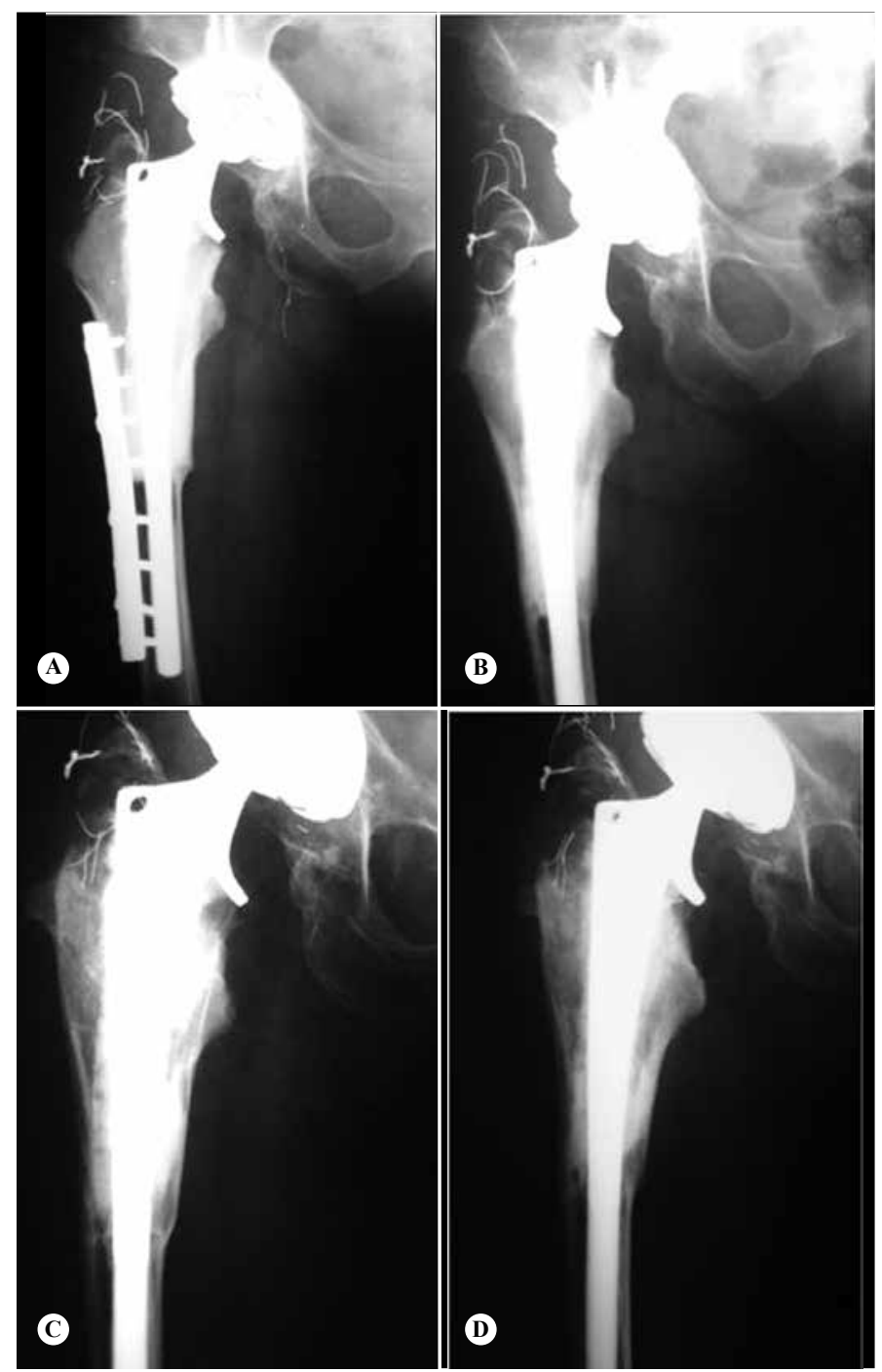

Figure 3 - (A) Radiograph prodı ced two years after the operation. (B) Radiograph produced seven years after the operation. (C) Radiograph produced 12 years after the operation. (D) Radiograph produced $17+1$ years after the operation.

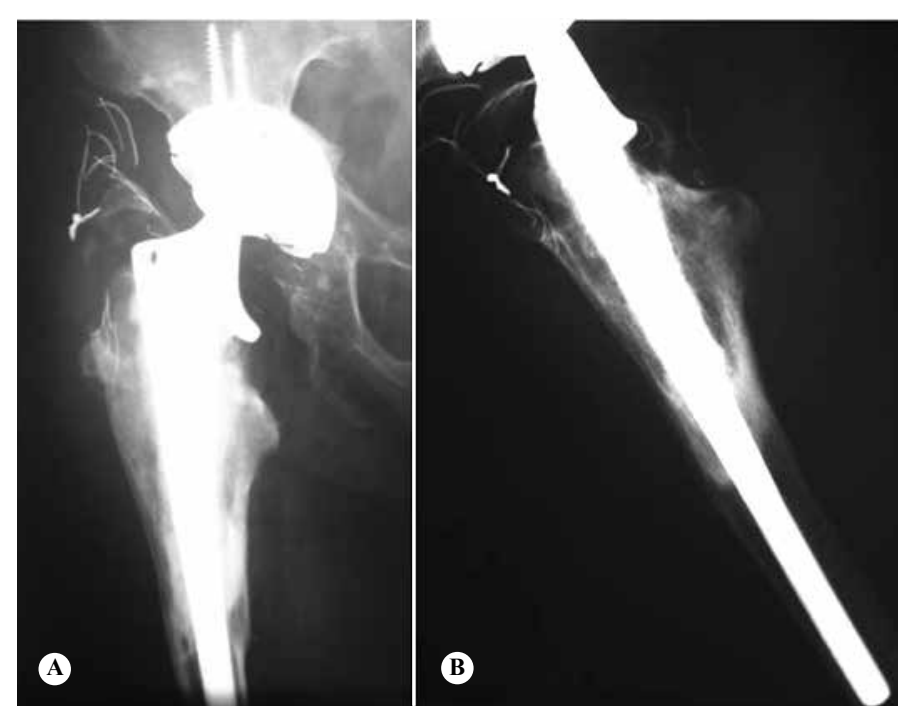

Figure 4 - Anteroposterior $(A P)$ radiograph $(A)$ and lateral radiogra$\mathrm{ph}(\mathrm{B})$ produced 21 years after the operation. femoral implant (Charnley prosthesis) was observed, associated with severe bone deficiency in the proximal third of the femur, thus characterizing a femoral defect of AAOS type $\mathrm{III}^{(4)}$. In relation to the acetabular implant, no signs of instability of the component were observed (Figure 5). The laboratory markers for infection were shown to be within the normal range.

The patient underwent THA revision, consisting of revision of the cemented acetabular component by means of an uncemented implant and use of a non-irradiated circumferential allograft from the proximal femur and a cemented femoral component, in association with stabilization of the graft with a plate and screws (Figure $6 \mathrm{~A}, \mathrm{~B}$ and $\mathrm{C}$ ).

After 12 years of follow-up, the Harris Hip Score ${ }^{(5)}$ was 78 points. Radiographically, it was observed that the implant was stable, with graft reabsorption in Gruen zone $\mathrm{II}^{(6)}$. The allograft had consolidated at the graft-host bone junction, and migration of the greater trochanter $(0.5 \mathrm{~cm})$ had occurred, with breakage of the cerclage threads (Figure $6 \mathrm{~A}, \mathrm{~B}$ and $\mathrm{C}$ ).

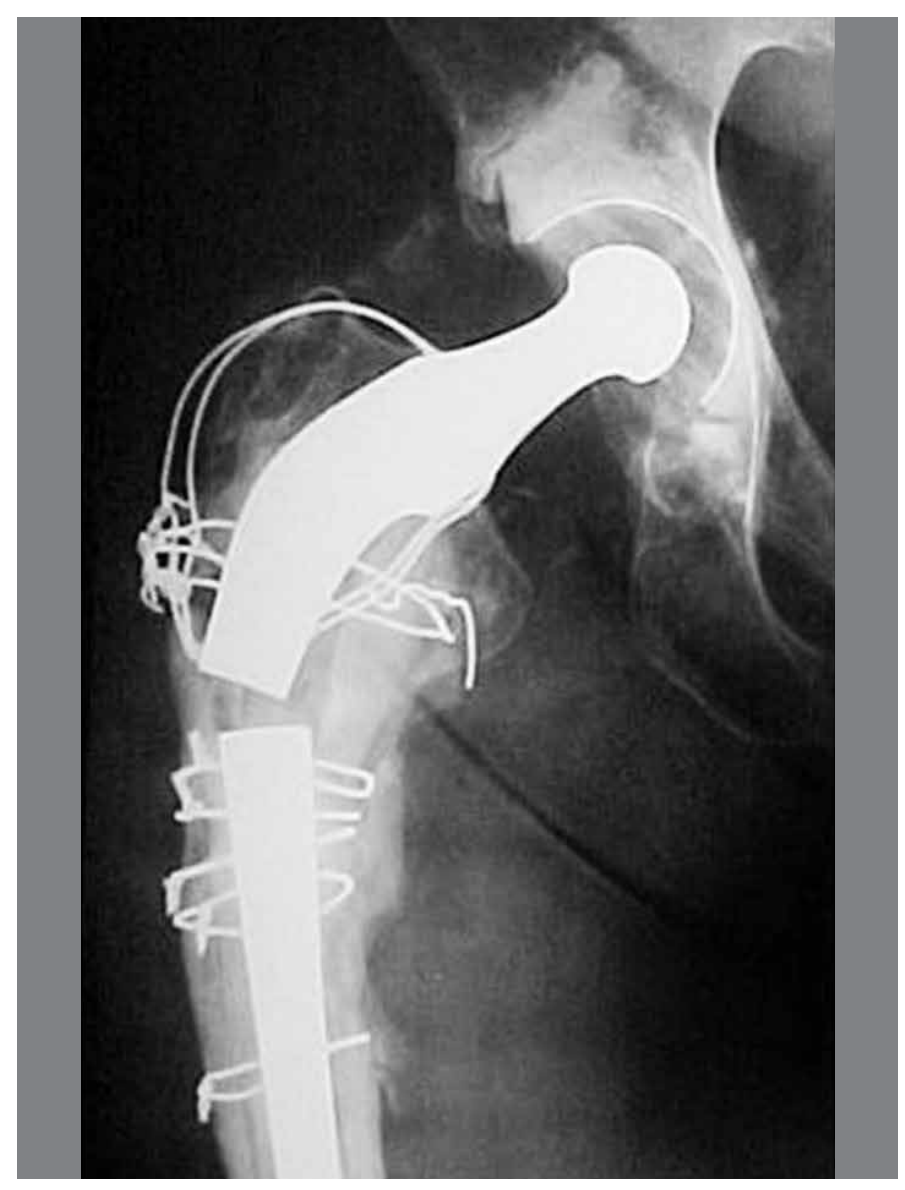

Figure 5 - Radiograph on 66-year-old female patient showing signs of loosening and fracturing of the femoral component, with AAOS type III femoral defect ${ }^{(4)}$. 
After 20 years of postoperative follow-up, the patient's general state was severely compromised due to complications from heart and lung disease. She was only walking in her home environment, without complaints of pain and, occasionally, with the aid of a support (walking frame). Radiographically, graft reabsorption was seen in Gruen zones I and $\mathrm{II}^{(6)}$, along with signs of instability of the femoral implant (pedestal sign) (Figure 7).

Twenty-one years after the surgical procedure, the patient died due to worsening of her clinical condition.

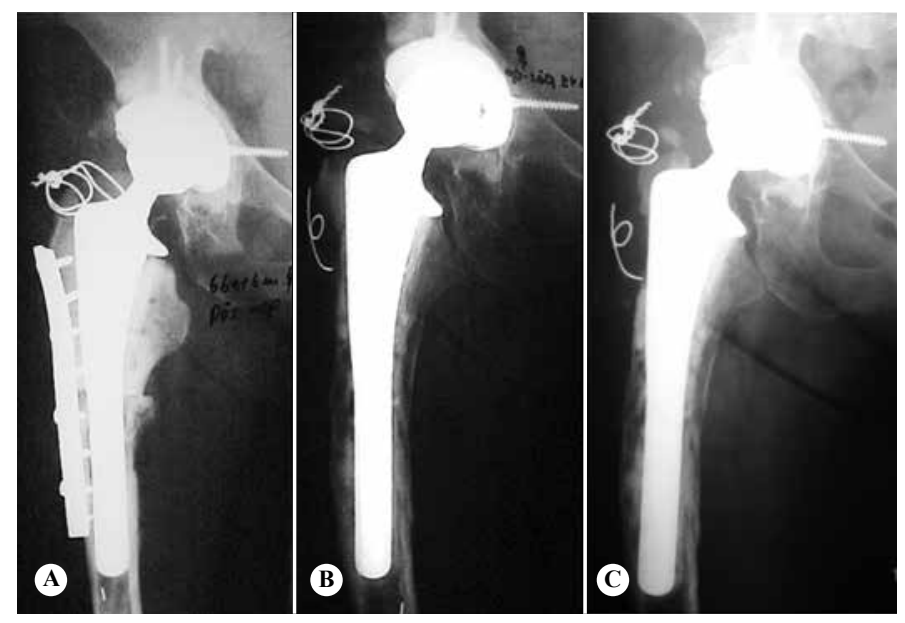

Figure 6 - (A) Radiograph produced during immediate postoperative period. (B) Radiograph produced $10+1$ years after the operation. (C) Radiograph produced $17+6$ years after the operation.

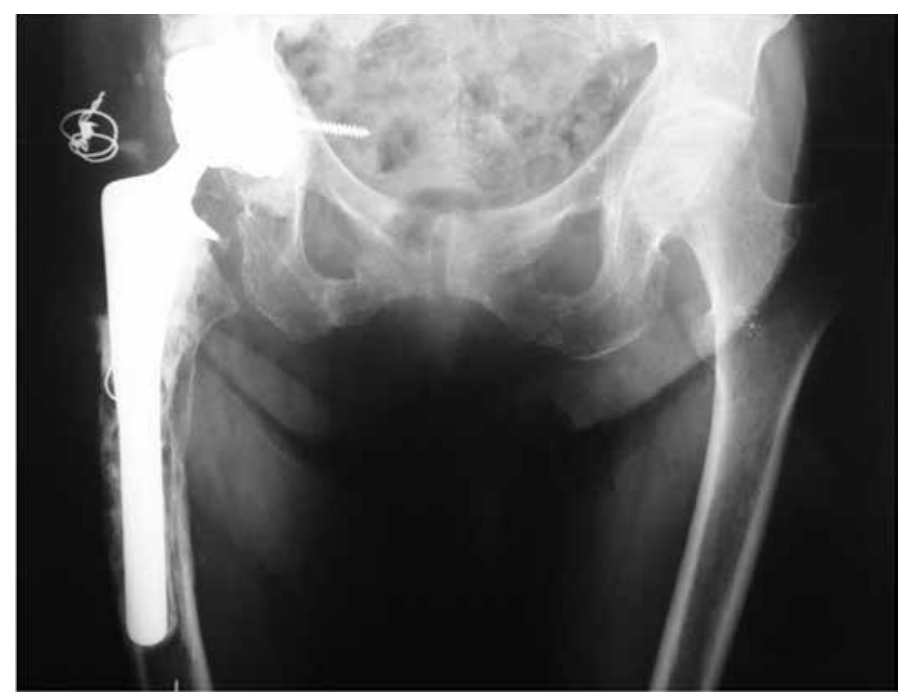

Figure 7 - Anteroposterior radiograph of the spleen, produced $20+2$ years after the operation.

\section{DISCUSSION}

Many surgical treatment methods for femoral reconstruction in cases of THA revision have been described in the literature. However, few option can be applied in cases of extensive circumferential bone defects (greater than $5 \mathrm{~cm})^{(3)}$. For these patients, the techniques most used were endoprostheses and circumferential femoral allografts.

Use of circumferential femoral allografts remains a matter of controversy in the literature. Many of the published papers on this topic have presented small numbers of patients, and a variety of surgical techniques and types of allografts have been used, as well as different implants. In addition, few of the authors have reported medium to long-term follow-ups ${ }^{(1-3,7-13)}$.

The indications for using femoral allografts in THA revision surgery are not well established. Gross and Hutchison ${ }^{(13)}$ recommended that they should be used in cases of circumferential femoral defects larger than $3 \mathrm{~cm}$, measured from the calcar, and in some combined defects and those with femoral discontinuity. These authors evaluated 63 hips that underwent femoral reconstruction using a circumferential femoral allograft in association with a cemented implant, and they observed that the reconstruction survived in $86 \%$, with a mean follow-up of 10 years. There were 13 complications ( $21 \%$ of the patients), which required new surgical interventions ${ }^{(3)}$.

Haddad et $\mathrm{al}^{(8)}$ reported that their hip reconstructions using circumferential proximal femoral allografts survived in $89 \%$ of the 55 hips evaluated, with a mean follow-up of 8.8 years. There was a large number of complications, including 22 cases of trochanteric pseudarthrosis and six cases of instability. However, their paper presented combined analysis on cases with cemented and uncemented implants.

Graham and Stockley ${ }^{(2)}$ presented the results from 25 hips that underwent femoral reconstruction using circumferential proximal femoral grafts and cemented implants, with two cases of aseptic loosening and one of infection. The mean length of follow-up was 4.3 years.

In the literature, a large number of complications relating to using this type of graft have been described, which is inherent to the severity and complexity of the cases ${ }^{(1-3,7-14)}$. The complications that most frequently required new surgical procedures are infection, pseudarthrosis, allograft fractures and instability. Another potential complication is graft reabsorption, which has been described in the literature in patients with medium to long-term follow-up ${ }^{(1-3,7-14)}$. In addition, use of homologous grafts presents a potential risk of disease transmission, which is minimal with 
the current methods for harvesting, processing and storing musculoskeletal tissues ${ }^{(15)}$.

Use of circumferential proximal femoral grafts allows correction of the hip abductor mechanism and leg length discrepancy, and also presents osteoconduc- tive potential in that is enables gradual substitution by host bone, with consequent replacement of the bone stock $^{(1-2)}$. For these reasons, this method should be taken into consideration in THA revision of cases of severe femoral defects, especially in young patients.

\section{REFERENCES}

1. Haddad FS, Garbuz DS, Masri BA, Duncan CP. Structural proximal femoral allografts for failed total hip replacements: a minimum review of five years. J Bone Joint Surg Br. 2000;82(6):830-6.

2. Graham NM, Stockley I. The use of structural proximal femoral allografts in complex revision hip arthroplasty. J Bone Joint Surg Br. 2004;86(3):337-43.

3. Blackley HR, Davis AM, Hutchison CR, Gross AE. Proximal femoral allografts for reconstruction of bone stock in revision arthroplasty of the hip. A nine to fifteen-year follow-up. J Bone Joint Surg Am. 2001;83(3):346-54.

4. D'Antonio J, McCarthy JC, Bargar WL, Borden LS, Cappelo WN, Collis DK, et al. Classification of femoral abnormalities in total hip arthroplasty. Clin Orthop Relat Res. 1993;(296):133-9.

5. Harris WH. Traumatic arthritis of the hip after dislocation and acetabular fractures: treatment by mold arthroplasty. An end-result study using a new method of result evaluation. J Bone Joint Surg Am. 1969;51(4):737-55.

6. Gruen TA, McNeice GM, Amstutz HC. "Modes of failure" of cemented stem-type femoral components: a radiographic analysis of loosening. Clin Orthop Relat Res. 1979;(141):17-27.

7. Duncan CP, Masri BA. Fractures of the femur after hip replacement. Instr Course Lect. 1995;44:293-304
8. Haddad FS, Spangehl MJ, Masri BA, Garbuz DS, Duncan CP. Circumferential allograft replacement of the proximal femur. A critical analysis. Clin Orthop Relat Res. 2000;(371):98-107.

9. Martin WR, Sutherland CJ. Complications of proximal femoral allografts in revision total hip arthroplasty. Clin Orthop Relat Res. 1993;(295):161-7.

10. Mankin HJ, Doppelt S, Tomford W. Clinical experience with allograft implantation. The first ten years. Clin Orthop Relat Res. 1983;(174):69-86.

11. Gitelis S, Piasecki P. Allograft prosthetic composite arthroplasty for osteosarcoma and other aggressive bone tumors. Clin Orthop Relat Res. 1991;(270):197-201.

12. Wang JW, Wang CJ. Proximal femoral allografts for bone deficiencies in revision hip arthroplasty: a medium-term follow-up study. J Arthroplasty. 2004;19(7):845-52.

13. Gross $A E$, Hutchison $C R$. Proximal femoral allografts for reconstruction of bone stock in revision hip arthroplasty. Orthopedics. 1998;21(9):999-1001.

14. Head WC, Berklacich FM, Malinin TI, Emerson RH Jr. Proximal femoral allografts in revision total hip arthroplasty. Clin Orthop Relat Res. 1987;(225):22-36.

15. Roos MV, Camisa Júnior A, Michelin AF. Procedimentos de um banco de ossos e aplicabilidade dos enxertos por ele proporcionados. Acta Ortop Bras. 2000;8(3):122-7. 